\title{
Efeito da ingestão de cafeína sobre os parâmetros da potência crítica
}

\author{
Effect of caffeine intake on critical power model parameters \\ determined on a cycle ergometer
}

1 Universidade Estadual de Campinas. Faculdade de Educação Física. Programa de Pós-Graduação em Educação Física. Campinas, SP. Brasil.

2 Universidade Estadual de Campinas. Faculdade de Educação Física. Laboratório de Atividades Aquáticas. Campinas, SP. Brasil.

3 Universidade Estadual de Campinas. Faculdade de Educação Física. Grupo de Estudo e Pesquisa em Sistema Neuromuscular. Campinas, SP. Brasil.

4 Universidade Estadual de Londrina. Centro de Educação Física e Desporto. Grupo de Estudo e Pesquisa em Metabolismo, Nutrição e Exercício. Londrina, PR. Brasil.

5 Universidade Federal do Rio Grande do

Norte. Departamento de Educação Física. Grupo de Estudo e Pesquisa em Biologia Integrativa do Exercício. Natal, RN. Brasil.

Recebido em 04/06/08 Revisado em 26/01/09 Aprovado em 18/05/09
Resumo - O objetivo do presente estudo foi verificar o efeito da ingestão de cafeína sobre os parâmetros do modelo de potência crítica determinado em cicloergômetro. Participaram do estudo oito sujeitos do sexo masculino. A ingestão de cafeína pura $\left(6 \mathrm{mg} \cdot \mathrm{kg}^{-1}\right)$ ou placebo (maltodextrina) foi realizada através de protocolo duplo-cego, 60 minutos antes dos testes. Para determinação da potência crítica, os sujeitos foram submetidos a quatro testes de cargas constantes em cicloergômetro, realizados aleatoriamente, nos momentos cafeína e placebo, nas intensidades de 80, 90, 100 e 110\% da potência máxima, a uma cadência de 70 rpm até a exaustão. A potência crítica e a capacidade de trabalho anaeróbio foram obtidas através de regressão não linear e ajuste de curva para o modelo hiperbólico potência-tempo. Para o tratamento estatístico, utilizaram-se-se o teste de Shapiro Wilk e o teste $t$ de Student pareado. Não foram encontradas diferenças significativas na potência crítica nas condições cafeína e placebo $(192,9 \pm 31,3$ vs 197,7 $\pm 29,4 \mathrm{~W}$, respectivamente). A capacidade de trabalho anaeróbio foi significativamente maior na condição cafeína $(20,1 \pm 5,2$ vs $16,3 \pm 4,2 \mathrm{~W}$, $\mathrm{p}<0,01)$. Foi, ainda, obtido um alto valor de associação $\left(\mathrm{r}^{2}\right)$ entre as condições cafeína e placebo $(0,98 \pm 0,02$ e 0,99 0,0). Através dos resultados obtidos, concluiu-se que a ingestão de cafeína não proporcionou melhorias no desempenho da potência crítica, entretanto, elevou os valores da capacidade de trabalho anaeróbio por influenciar o desempenho nas cargas de maior intensidade e menor duração.

Palavras-chave: Potência Crítica; Cafeína; Ergogênico.

Abstract - The aim of this study was to evaluate the effect of caffeine intake on critical power model parameters determined on a cycle ergometer. Eight male subjects participated in this study. A double-blind protocol consisting of the intake of pure caffeine $(6 \mathrm{mg} / \mathrm{kg}$ ) or placebo (maltodextrin) $60 \mathrm{~min}$ before testing was used. Subjects were submitted to four constant-load tests on a cycle ergometer. These tests were conducted randomly in the caffeine and placebo groups [checar] at intensities of 80, 90, 100 and $110 \%$ maximum power at a rate of $70 \mathrm{rpm}$ until exhaustion to determine the critical power. As a criterion for stopping the test was adopted any rate fall without recovery by more than five seconds. The critical power and anaerobic work capacity were obtained by nonlinear regression and fitting of the curve to a hyperbolic power-time model. The ShapiroWilk test and paired Student t-test were used for statistical analysis. No significant differences in critical power were observed between the caffeine and placebo groups $(192.9 \pm 31.3$ vs $197.7 \pm$ $29.4 \mathrm{~W}$, respectively). The anaerobic work capacity was significantly higher in the caffeine group $(20.1 \pm 5.2$ vs $16.3 \pm 4.2 \mathrm{~W}, p<0.01)$. A high association $\left(r^{2}\right)$ was observed between the caffeine and placebo conditions $(0.98 \pm 0.02$ and $0.99 \pm 0.0$, respectively). We conclude that caffeine intake did not improve critical power performance but increased anaerobic work capacity by influencing performance at loads of higher intensity and shorter duration.

Key words: Critical power; Caffeine; Ergogenic; Ergogenic supplement. 


\section{INTRODUÇÃO}

O efeito ergogênico da cafeína está bem documentado para eventos de resistência, observando-se aumento significativo no tempo de exaustão e na produção média de potência ${ }^{1-4}$. Para descrever a sequência de acontecimentos responsáveis pela melhoria do desempenho em exercícios físicos prolongados, alguns estudos apontam que a cafeína poderia causar aumento na oxidação de ácidos graxos livres (AGLs) o que reduziria a utilização de carboidratos $(\mathrm{CHO})$, sendo este último considerado um dos principais responsáveis pela instauração da fadiga ${ }^{1,5,6}$.

Estudos também evidenciam que a cafeína poderia exercer influência sobre o desempenho em atividades de alta intensidade e curta duração ${ }^{7-9}$. A teoria melhor aceita, atualmente, para descrever seus efeitos sobre o metabolismo anaeróbio, pressupõe uma ação direta da cafeína sobre coprodutos do músculo esquelético. As possibilidades incluem: alteração de íons, particularmente, sódio e potássio; inibição da fosfodiesterase (PDE), possibilitando um aumento na concentração de adenosina monofosfato cíclica (AMPc); e aumento na mobilização de cálcio através do retículo sarcoplasmático, com consequente aumento dos níveis intracelulares de cálcio nos músculos, aumentando a eficiência da contração muscular ${ }^{1,10}$. Embora os mecanismos responsáveis pelos efeitos ergogênicos da cafeína ainda não estejam esclarecidos ${ }^{2,11}$, acredita-se que a potência crítica (PC), por fornecer parâmetros sobre a capacidade aeróbia e anaeróbia, possa ser adequada para verificar sua ação sobre estes metabolismos.

A PC é um conceito teórico, sugerido na década de 60 por Monod \& Scherrer ${ }^{12}$. Segundo esses autores, a PC seria o limite superior de esforço sustentável sem a ocorrência de fadiga. Através deste conceito, determina-se, também, a capacidade de trabalho anaeróbio (CTAn) que representa o trabalho total agregado, realizado pelas reservas limitadas de energia do corpo (fosfatos e glicólise) ${ }^{12}$. Diversos estudos procuraram relacionar a PC com diferentes variáveis aeróbias e observaram altas correlações entre este parâmetro e o limiar anaeróbio ${ }^{13,14} \mathrm{e}$ com diferentes limiares de fadiga $\left(\mathrm{La}_{\mathrm{FT}}\right.$, $\mathrm{iEMG}_{\mathrm{FT}}, \mathrm{VO}_{2 \mathrm{FT}}$ e $\left.\mathrm{HR}_{\mathrm{FT}}\right)^{15,16}$. A CTAn por sua vez, tem apresentado alta correlação com máximo déficit acumulado de oxigênio ${ }^{17}$. $\mathrm{O}$ modelo da PC foi baseado na relação hiperbólica entre a potência realizada $\left(W_{\text {lim }}\right)$ e seu respectivo tempo de exaustão $\left(t_{\text {lim }}\right)$. Frequentemente esta relação é expressa através da equação $t=C T A n /(W-P C)$, onde a PC é a assíntota da taxa de trabalho e a CTAn é o grau de curvatura da relação ${ }^{18}$.

Apesar das inúmeras pesquisas, abordando o efeito da ingestão da cafeína, seu potencial ergogênico ainda não está completamente elucidado. Assim sendo, o objetivo do presente estudo foi investigar o efeito da ingestão de cafeína sobre os parâmetros do modelo de Potência Crítica determinados em cicloergômetro.

\section{PROCEDIMENTOS METODOLÓGICOS}

\section{Sujeitos}

Participaram voluntariamente deste estudo oito homens (25,75 $\pm 3,41$ anos; $82,00 \pm 9,12 \mathrm{~kg} ; 180,25$ $\pm 5,44 \mathrm{~cm}$ ), saudáveis e fisicamente ativos. Todos os sujeitos, após receberem informações sobre as finalidades do estudo e os procedimentos aos quais seriam submetidos, assinaram termo de consentimento livre e esclarecido.

Este estudo foi aprovado pelo Comitê de Ética em Pesquisa, de acordo com as normas da Resolução 196/96 do Conselho Nacional de Saúde sobre pesquisa, envolvendo seres humanos, sob o número 228/2005 (Universidade Estadual de Campinas).

\section{Protocolo experimental}

Todos os sujeitos foram submetidos a duas condições experimentais, em momentos distintos, através de procedimento duplo-cego: a) suplementação com cafeína pura (CAF) (6mg.kg $\left.{ }^{-1}\right)$; b) suplementação com maltodextrina (PLA). Adotou-se o repouso de sete dias entre as condições para uma completa recuperação dos participantes do experimento.

Ingestão de cafeína e placebo

Os indivíduos ingeriram CAF ou PLA, sessenta minutos antes do início dos testes de cargas contínuas $\left(\mathrm{W}_{\text {lim }}\right)$, realizados para a determinação da potência crítica (PC), permanecendo em repouso do momento da ingestão até o início do teste para possibilitar a completa absorção.

\section{Teste incremental máximo}

O teste incremental máximo $\left(\mathrm{TI}_{\max }\right)$ foi realizado em cicloergômetro eletromagnético (Corival 400, Quinton Instruments). Os participantes realizaram aquecimento padronizado, consistindo de três minutos a 50 watts (W) e 70 rotações por minuto (rpm). O TI $\max _{\text {in }}$ inciou-se com carga de $0 \mathrm{~W}$ e 70 rpm, sendo incrementados $20 \mathrm{~W}$, a cada minuto, até a exaustão voluntária. Como critério de interrupção do teste, adotou-se a queda na cadência 
de revoluções sem recuperação, por mais de cinco segundos. $\mathrm{O} \mathrm{TI}_{\max }$ foi utilizado para determinar a mais alta carga de trabalho mantida pelo indivíduo por, pelo menos, 30 segundos $\left(\mathrm{W}_{\max }\right)$.

\section{Determinação da potência crítica} e da capacidade de trabalho anaeróbio

A potência crítica (PC) foi determinada nos dois momentos do experimento (CAF e PLA) através de quatro cargas constantes $\left(\mathrm{W}_{\text {lim }}\right)$, com intensidades de $80 \%, 90 \%, 100 \%$ e $110 \%$ da $W_{\text {max }}$ e cadência de 70 rpm até a exaustão voluntária (critério de interrupção do teste como descrito no $\mathrm{TI}_{\max }$. As cargas constantes foram realizadas de forma aleatória, com intervalo de 72 horas entre elas.

A equação abaixo relaciona a potência/carga (W) durante cada um dos testes de $\mathrm{W}_{\text {lim }}$ com a duração dos mesmos, por meio de uma hipérbole. A assíntota, no ponto em que toca o eixo $\mathrm{X}$, representa a potência crítica (PC) em W, e o grau de curvatura da hipérbole constitui a estimativa da capacidade de trabalho anaeróbio (CTAn) (Figura 1) $)^{15}$.

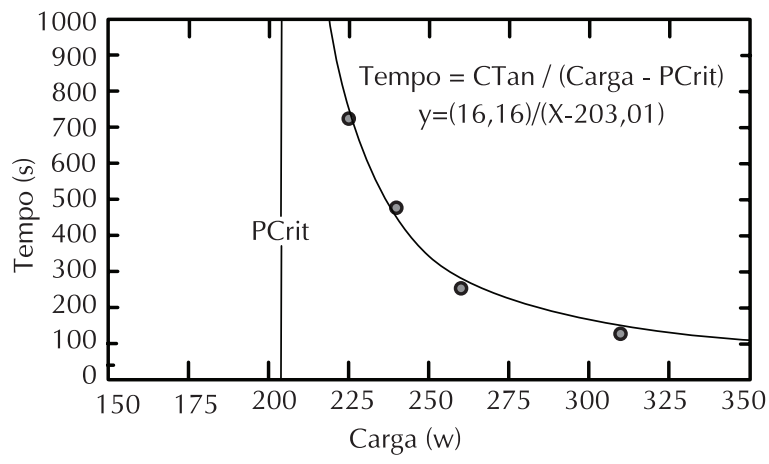

Figura 1. Determinação da PC e CTAn de um indivíduo por meio de análise de regressão não linear e ajuste de curva para o modelo hiperbólico potência - tempo.

A PC e a CTAn foram obtidas através de regressão não linear, utilizando o software STATISTICA 6.0 ${ }^{\mathrm{TM}}$. Também foi estimado o coeficiente de determinação $\left(\mathrm{r}^{2}\right)$ relacionado ao ajuste dos dados experimentais à equação.

Em todas as coletas a temperatura ambiente foi mantida entre 21 e $24^{\circ} \mathrm{C}$. Os sujeitos foram testados sempre na mesma hora do dia para minimizar os efeitos da variação biológica diurna. Os mesmos foram orientados, ainda, para que não realizassem atividades físicas vigorosas no período do experimento. Também foram orientados a não consumirem bebidas alcoólicas e substâncias cafeinadas nas $24 \mathrm{~h}$ precedentes a cada teste. Para a familiarização com os protocolos de testes e equipamentos utilizados, os voluntários foram submetidos a estudo piloto.

\section{Tratamento estatístico}

Após constatação da normalidade dos dados através do teste de Shapiro Wilk, os valores médios da PC, CTAn, e do $r^{2}$, nas condições CAF e PLA, foram confrontados mediante aplicação do teste "t" de Student pareado. Todas as informações foram processadas no pacote computadorizado STATIS. TICA 6.0 ${ }^{\mathrm{TM}}$ (STATSOFT ${ }^{\circledR}$, USA). O nível de significância adotado para as análises foi $P<0,05$.

\section{RESULTADOS}

A Tabela 1 apresenta os valores médios e desvios padrão em W obtidos no teste incremental máximo $\left(\mathrm{TI}_{\mathrm{Max}}\right)$ e nos testes de cargas constantes $\left(\mathrm{W}_{\text {lim }}\right)$ a $80,90,100$ e $110 \%$ da $W_{\text {max }}$ utilizadas na determinação da PC e da CTAn.

Tabela 1. Resultado em média e desvio-padrão (DP) do teste incremental máximo ( $\mathrm{TI}_{\max }$ ) e dos testes com cargas constantes $\left(W_{\lim }\right)$ a $80,90,100$ e $110 \%$ da $W_{\max }$ utilizados na determinação da PC e CTAn $(n=8)$.

\begin{tabular}{lcc}
\hline Variáveis & Média $(\mathrm{W})$ & $\mathrm{DP}$ \\
\hline $\mathrm{TI}_{\max }$ & 280,00 & 30,5 \\
$\mathrm{~W}_{\text {lim }} 80 \%$ & 212,63 & 23,53 \\
$\mathrm{~W}_{\lim } 90 \%$ & 240,38 & 27,36 \\
$\mathrm{~W}_{\lim } 100 \%$ & 268,50 & 33,63 \\
$\mathrm{~W}_{\lim } 110 \%$ & 301,50 & 31,73 \\
\hline
\end{tabular}

Na Tabela 2, são apresentados os valores médios dos tempos de exaustão $\left(t_{\text {lim }}\right)$ correspondentes às potências $\left(\mathrm{W}_{\text {lim }}\right)$ utilizadas para o cálculo da PC e da CTAn nas condições CAF e PLA. As cargas utilizadas (80, 90, 100 e 110\%) foram determinadas de forma que a duração mínima até a exaustão nos testes fosse de, aproximadamente, de três minutos ${ }^{18}$.

A condição suplementado com CAF foi significativamente maior que o PLA nas cargas com maiores intensidades e menores durações $(280 \pm$ $80,85 \mathrm{~W}$ vs $251,38 \pm 74,99 \mathrm{~W}$ e $187 \pm 40,87 \mathrm{~W}$ vs $160,75 \pm 41,62 \mathrm{~W}$, respectivamente para $\mathrm{W}_{\lim } 100$ e $\left.\mathrm{W}_{\lim } 110 \%\right)$.

Os resultados da PC, CTAn e do $r^{2}$, nas condições CAF e PLA, são apresentados na tabela 3. Os valores da PC e do $r^{2}$ não foram significativamente diferentes entre as condições CAF e PLA. Em contrapartida, o valor da CTAn foi significativamente maior na condição CAF quando comparado a PLA.

\section{DISCUSSÃO}

Foram encontradas diferenças significativas entres as condições CAF e PLA durante as cargas mais altas 
Tabela 2. Valores médios ( \pm DP) dos tempos de exaustão $\left(t_{\text {lim }}\right)$ correspondentes às cargas constantes $\left(W_{\text {lim }}\right)$ de $80,90,100$ e $110 \%$ da $W_{\max }$ utilizadas para o cálculo da PC e da CTAn nas condições cafeína (CAF) e placebo (PLA) $(n=8)$.

\begin{tabular}{ccccccccc}
\hline & $\mathrm{W}_{\lim } 80 \%$ & & $\mathrm{~W}_{\lim } 90 \%$ & & $\mathrm{~W}_{\lim } 100 \%$ & & \multicolumn{2}{c}{$\mathrm{W}_{\lim } 110 \%$} \\
\hline & $\mathrm{CAF}$ & $\mathrm{PL}$ & $\mathrm{CAF}$ & $\mathrm{PL}$ & $\mathrm{CAF}$ & $\mathrm{PL}$ & $\mathrm{CAF}$ & $\mathrm{PL}$ \\
\multirow{2}{*}{$\mathrm{t}_{\lim }(\mathrm{seg})$} & $1089,00 \pm$ & $1059,86 \pm$ & 468,00 & 429,71 & $280,00^{*}$ & 251,38 & $187,63^{*}$ & 160,75 \\
& 288,46 & 254,42 & $\pm 139,55$ & $\pm 68,02$ & $\pm 80,85$ & $\pm 74,99$ & $\pm 40,87$ & $\pm 41,62$ \\
\hline
\end{tabular}

*Diferença significativa entre as condições CAF e PLA $(P<0,01)$.

Tabela 3. Valores médios ( \pm DP) da potência crítica $(P C)$ da capacidade de trabalho anaeróbio (CTAn) e do coeficiente de determinação $\left(r^{2}\right)$ nas condições cafeína (CAF) e placebo (PLA) $(n=8)$.

\begin{tabular}{lcccc}
\hline Parâmetros & CAF & PLA & T & P \\
\hline PC (W) & $192,91 \pm 31,34$ & $197,78 \pm 29,45$ & $-1,73$ & 0,13 \\
CTAn (KJ) & $20,10 \pm 5,23$ & $16,31 \pm 4,28$ & 3,92 & $<0,01$ \\
$r^{2}$ & $0,98 \pm 0,02$ & $0,99 \pm 0,01$ & $-0,24$ & 0,82 \\
\hline
\end{tabular}

$\mathrm{W}_{\lim } 100$ e $\mathrm{W}_{\lim } 110 \%(280,00 \pm 80,85 \mathrm{~W}$ ws $251 \pm$ $74,99 \mathrm{~W}$ e $187,63 \pm 40,87 \mathrm{~W}$ vs $160,75 \pm 41,62 \mathrm{~W}$ respectivamente). Esse melhor desempenho durante as cargas retangulares mais intensas não afetou o valor da PC $(192,92 \pm 31,34 \mathrm{~W}$ vs 197,78 \pm 29,45 W; condições CAF e PLA respectivamente). Contudo, aumentou significativamente os valores da CTAn na condição CAF $(20,10 \pm 5,23 \mathrm{~W}$ vs $16,31 \pm 4,28$ $\mathrm{W}, \mathrm{P}<0,01)$. Housh et $\mathrm{a}^{19}$ apontam que a PCé altamente influenciada por cargas de menor intensidade, obtendo-se com isso maior tempo de duração do exercício até a exaustão. A CTAn, no entanto, é altamente sensível à manipulação do seu principal componente energético, o glicogênio muscular ${ }^{20}$.

A sensibilidade da CTAn a manipulações dietéticas já foi demonstrada em estudos realizados com creatina monoidratada. A CTAn apresentou aumento significativo após a suplementação, não exercendo qualquer influência sobre a $\mathrm{PC}^{21,22}$. Esse comportamento também foi demonstrado após treinamento intenso com característica anaeróbia, sendo verificado aumento significativo na CTAn, sem modificações na PC. Contudo, quando a característica do treinamento passou a ser aeróbia, observou-se um efeito contrário, com aumento da PC, sem qualquer alteração na CTAn ${ }^{23,24}$.

De acordo com os resultados obtidos no presente estudo, a ingestão de $6 \mathrm{mg} \cdot \mathrm{kg}^{-1}$ de cafeína resultou em melhora na CTAn de, aproximadamente, 8,1\%, quando comparada com o grupo PLA. Estes resultados estão de acordo com estudo realizado por Anselme et $\mathrm{a}^{25}$ que reportaram que a ingestão de cafeína aumentou em aproximadamente $7 \%$ a potência anaeróbia máxima. Também proporcionou aumento de, aproximadamente, 4-6\% na velocidade de nadadores treinados durante sprint de 100 metros $^{26}$.

Jackman et $\mathrm{al}^{27}$ concluíram que a ingestão de cafeína pode resultar em aumento da resistência muscular durante exercício intenso que leve a fadiga em 5 minutos, após a ingestão de $6 \mathrm{mg} \cdot \mathrm{Kg}^{-1}$ de cafeína. Mais recentemente, estudo examinando o desempenho em corrida de alta intensidade (3-4 min) observou melhora significante no déficit máximo de oxigênio acumulado e no tempo de exaustão após ingestão de cafeína ${ }^{28}$. Da mesma forma, Bell et $\mathrm{al}^{29}$ constataram melhora significativa no déficit máximo de oxigênio acumulado e no tempo de exaustão em cicloergômetro após ingestão de cafeína. Esses resultados têm implicações importantes, uma vez que a melhoria do desempenho físico demonstrou estar relacionada a uma possível ação ergogênica da cafeína sobre a capacidade anaeróbia. Contudo, no presente estudo, não foram observadas melhorias significativas sobre a PC ou sobre as cargas preditivas de intensidades mais baixas $\left(\mathrm{W}_{\text {lim }} 80\right.$ e 90\%). Achados semelhantes foram relatados por alguns estudos realizados em cicloergômetro com intensidade entre 75 e $85 \%$ do $\mathrm{VO}_{2 \text { máx }} 30,31$. Assim como nesse estudo, as pesquisas acima citadas foram realizadas com sujeitos não treinados. Este fator é apontado como de grande influência para atividades vigorosas e de longa duração ${ }^{31}$.

Pelo nosso conhecimento, esta é a primeira investigação que aborda a suplementação de cafeína sobre os parâmetros do modelo da PC. Apesar da PC ser considerada um indicador sensível a variações de estímulos e treinamento, ressalta-se que o nível de treinamento dos sujeitos pode interferir no seu resultado, sendo este considerado um fator limitante desta pesquisa.

\section{CONCLUSÃO}

A ingestão de cafeína mostrou-se eficaz na manipulação da CTAn, considerada o componente anaeróbio do modelo da PC. Este fato se deve a 
melhoria no desempenho das $\mathrm{W}_{\text {lim }}$ de menor duração e de mais altas intensidades. Contudo, a CAF não apresentou melhorias no resultado da $\mathrm{PC}$, uma vez que seu valor sofre influência das cargas com durações maiores.

\section{Agradecimentos}

Os autores agradecem ao Conselho Nacional de Desenvolvimento Científico e Tecnológico (CNPq), a Fundação de Amparo a Pesquisa do Estado de São Paulo (FAPESP), e a Coordenação de Aperfeiçoamento de Pessoal de Nível Superior (CAPES), pelas bolsas outorgadas.

\section{REFERÊNCIAS BIBLIOGRAFICAS}

1. Davis JM, Zhao Z, Stock HS, Mehl KA, Buggy J, Hand GA. Central nervous system effects of caffeine and adenosine on fatigue. Am J Physiol 2003; 284 (Suppl):R399-R404.

2. Graham TE. Caffeine and exercise: metabolism, endurance and performance. Sports Med 2001;31(11):785-807.

3. Lindinger MI, Graham TE, Spriet LL. Caffeine attenuates the exercise-induced increases in plasma $\left[\mathrm{K}^{+}\right]$in humans, J Appl Physiol 1993;74(3):1149-1155.

4. Tarnopolsky MA, Cupido C. Caffeine potentiates low frequency skeletal muscle force in habitual and nonhabitual caffeine users. J Appl Physiol 2000;89(5):1719-1724.

5. Graham TE, Rush JW, Van Soeren MH. Caffeine and exercise: metabolism and performance. Can J Appl Physiol 1994;19(2):111-138.

6. Greer F, Friars D, Graham TE. Comparison of caffeine and theophylline ingestion: exercise metabolism and endurance. J Appl Physiol 2000;89(5):1837-1844.

7. Bruce CR, Anderson ME, Fraser SF, Stepto NK, Klein R, Hopkins WG, et al. Enhancement of 2000-m rowing performance after caffeine ingestion. Med Sci Sports Exerc 2000; 32(11):1958-1963.

8. Doherty M, Smith PM, Hughes MG, Davison RC. Caffeine lowers percentual response and increases power output during high-intensity cycling. J Sports Sci 2004;22(7):637-643.

9. Jackman M, Wendling P, Friars D, Graham TE. Metabolic, catecholamine and endurance responses to caffeine during intense exercise. J Appl Physiol 1996;81(4):1658-1663.

10. Spriet LL. Caffeine and performance. Int J Sports Nutr 1995;5(Suppl):S84-S99.

11. Kalmar JM, Caffarelli E. Caffeine: a valuable tool to study central fatigue in humans? Exerc Sport Sci Rev 2004;32(4):143-147.

12. Monod H, Sherrer J. The work capacity of a synergic muscular group. Ergonomics 1965;8(3):329-338.
13. Wakayoshi K, Ilkuta K, Yoshida T. Determination and validity of critical velocity speed as an index of swimming performance in the competitive swimmer. Eur J Appl Physiol Occup Physiol 1992;64(2):153-157.

14. Wakayoshi K, Yoshida T, Udo M, Harada T, Moritani T, Mutoh Y, et al. Does critical swimming velocity represent exercise intensity at maximal lactate steady state? Eur J Appl Physiol Occup Physiol 1993;66(1):90-95.

15. Moritani T, Nagata A, Devries H, Muro M. Critical power as a measure of physical work capacity and anaerobic threshold. Ergonomics 1981;24(5):339-350.

16. Le Chevalier JM, Vandewalle H, Thépaut-Mathieu C, Stein JF, Caplan L. Local critical power is an index of local endurance. Eur J Appl Physiol 2000;81(1-2):120-127.

17. Hill DW, Smith JC. A method to ensure the accuracy of estimates of anaerobic capacity derived using the critical power concept. J Sports Med Phys Fitness 1994;34(1):23-37.

18. Calis JF, Denadai BS. Influência das cargas selecionadas na determinação da potência crítica determinada no ergômetro de braço em dois modelos lineares. Rev Bras Med Esporte 2000;6(1):1-4.

19. Housh DJ, Housh TJ, Bauge SM. A methodological consideration for the determination of critical power and anaerobic work capacity. Res Quarterly Exerc Sport 1990;61(4):406-409.

20. Miura A, Sato H, Whipp BJ, Fukuba Y. The effect of glycogen depletion on the curvature constant parameter of the power-duration curve for cycle ergometry, Ergonomics 2000; 46(1):133-141.

21. Smith JC, Stephens DP, Hall EL, Jackson AW, Earnest CP. Effect of oral creatine ingestion on parameters of work-time relationship and time to exhaustion in high-intensity cycling. Eur J Appl Physiol 1998;77(4):360-365.

22. Eckerson JM, Stout JR, Moore GA, Stone NJ, Nishimura K, Tamura K. Effect of two and five days of creatine loading on anaerobic working capacity in women. J Strength Condit Res 2004;18(1):168-173.

23. Jenkins DG, Quigley BM. Endurance training enhances critical power. Med Sci Sports Exerc 1992;24(11):1283-1289.

24. Jenkins DG, Quigley BM. The influence of high-intensity exercise training on the Wlin-Tlim relationship. Med Sci Sports Exerc 1993;25(2):275-282.

25. Anselme F, Collomp K, Mercier B, Ahmaidi S, Prefaut C. Caffeine increases maximal anaerobic power and blood lactate concentration. Eur J Appl Physiol 1992;65(2):188-191.

26. Paton CD, Hopkins WG, Volebregt L. Little effect of caffeine ingestion on repeated sprints in team-sport athletes. Med Sci Sports Exerc 2001;33(5):822-825.

27. Jackman M, Wendling A, Friars D, Graham TE. Metabolic, catecholamine, and endurance responses to caffeine during intense exercise. J Appl Physiol1996;81(4):1658-1663.

28. Doherty M. The effects of caffeine on the maximal accumulated oxygen deficit and short-term running performance. Int J Sports Nutr 1998;8(2):95-104. 
29. Bell DG, Jacobs I, Ellerington K. Effect of caffeine and ephedrine ingestion on anaerobic exercise performance. Med Sci Sports Exerc 2001;33(11):1399-1403.

30. Alves MN, Ferrari-Auarek WM, Pinto KMC, Sá KR, Viveiros JP, Pereira HAA, et al. Effects of caffeine on tryptophan on rectal temperature, metabolism, total exercise time, rate of perceived exertion and heart rate. Braz J Med Biol Res 1995;28(6):705-709.

31. Butts NK, Crowell D. Effect of caffeine ingestion on cardiorespiratory endurance in men and women. Res Q Exerc Sport 1985;56(4):301-305.
Endereço para correspondência Marcus Vinicius Machado Instituto Oswaldo Cruz Laboratório de Investigação Cardiovascular Av. Brasil, 4365, Manguinhos.

CEP 21045-900 - Rio de Janeiro, RJ. Brasil. E-mail: marcus_machado@globomail.com 